\title{
Minimally Invasive Transforaminal Lumbar Interbody Fusion for the Surgical Management of Post-Discectomy Syndrome
}

\author{
Ahmed Bahaa Al Din AlShazli ${ }^{1}$, Ashraf Yassin Amer ${ }^{1}$, Ahmed Maher Sultan ${ }^{2}$, \\ Ahmed Samir Barakat ${ }^{2}$, Wael Koptan ${ }^{2}$, Yasser ElMiligui ${ }^{2}$, Hesham Shaker ${ }^{1}$ \\ ${ }^{1}$ Agouza Armed Forces Spine Surgery Center, Giza, Egypt \\ ${ }^{2}$ Orthopedics and Traumatology Department, Faculty of Medicine, Cairo University, Cairo, Egypt
}

Study Design: This was a prospective case series of 30 patients with post-discectomy syndrome with an average of 18 months of follow-up (level IV).

Purpose: The efficacy of post-discectomy syndrome managed by minimally invasive surgery transforaminal lumbar interbody fusion (MIS-TLIF) was evaluated.

Overview of Literature: In post-discectomy syndrome wherein conservative treatment had failed, the best surgical treatment modality still remains controversial.

Methods: Patients were functionally assessed using the Visual Analog Scale (VAS) for low back pain (LBP) and leg pain (LP) and Oswestry Disability Index (ODI). Radiological fusion was confirmed with plain X-rays and when indicated with computed tomography scan at 12 months postoperatively. A total of 30 patients with 37 operated at lumbar levels with failed discectomy surgery who met our inclusion criteria were treated with MIS-TLIF.

Results: The ODI of all patients showed significant improvement from a mean of $73.78 \%$ preoperatively to $16.67 \%$ at 1 month and $14.13 \%$ at 12 months postoperatively. The preoperative LBP VAS score (mean, 4.37) showed a significant decrease $(p<0.001)$ to 1.90 at 1 month and 1.10 at 12 months. Preoperative LP VAS score of limb pain averaged 7.53 and showed a significant $(p<0.001)$ decrease to 3.47 at 1 month and 1.10 at 12 months. All patients attained radiological fusion at 12 months.

Conclusions: MIS-TILF constitutes a valid and effective treatment option for patients with post-discectomy syndrome.

Keywords: Post-diskectomy syndrome; Failed back surgery; Recurrent disc prolapse; Interbody fusion; Minimally invasive surgery transforaminal lumbar interbody fusion

\section{Introduction}

Post-discectomy syndrome describes the presence of persistent disabling pain in the lumbar spine, hip, or lower limb after primary discectomies and is reported to occur in $5 \%-18 \%$ cases [1]. Early failure is generally due to poor patient selection, improper procedure, or infection (discitis). A new onset of pain following good surgical results

Received May 1, 2019; Revised Jun 12, 2019; Accepted Jun 27, 2019

Corresponding author: Ahmed Samir Barakat

Orthopedic Surgery Department, Cairo University Hospitals, Kasr Alainy Street, Cairo 11562, Egypt

Tel: +20-11-0273-2222, Fax: +20-2-23644383, E-mail: ahmedsamir222222@live.com 
may be due to recurrent disc herniation, instability of the operated segment, or affection at a different disc level $[2,3]$.

Postoperative complications should be aggressively treated from the start [4]. Serial investigations are required for the appropriate diagnosis of post-discectomy syndrome, and conservative treatment might be effective in early diagnosed cases [5]. Conditions amenable to conservative treatment include epidural fibrosis, arachnoiditis, pedicle and certain body fractures, psychogenic factors, early discitis, extensive multilevel disc degeneration, sacroiliac disease, and peripheral nerve syndrome [6]. After the failure of conservative treatment, revision discectomy or fusion of the affected disc level is indicated $[7,8]$. The conventional modalities of surgical treatment are as follows: re-discectomy, posterior lumbar interbody fusion (PLIF), transforaminal lumbar interbody fusion (TLIF), anterior lumbar interbody fusion, and decompression with posterolateral fusion. These modalities have shown various complications, and most of them are related to the adhesions and scar tissue dissection, which might lead to dural tears, root avulsion, and related consequences [6].

Minimally invasive TLIF (MIS-TLIF) appears to achieve high rates of arthrodesis while minimizing iatrogenic soft tissue/muscle injury and blood loss. Short hospital stays and decreased narcotic requirements are the advantages of MIS-TLIF [6,9]. However, MIS-TLIF is technically more challenging than open procedures that require a steep learning curve and more expensive resources [10].

This study aimed to assess the functional outcome of MIS-TLIF using the Oswestry Disability Index (ODI) and Visual Analog Scale (VAS) of low back and lower limb pain and the radiological outcome regarding osseous union within the first postoperative year.

\section{Materials and Methods}

This prospective case series included 30 patients who underwent MIS-TLIF through a percutaneous posterior lumbar pedicular screw fixation, microscopic minimally invasive transforaminal revision discectomy, and interbody cage fusion surgery for post-discectomy. This study was performed at Agouza Armed Forces Center for Spine Surgery, Agouza, Giza, Egypt, from October 2015 to 2017. The average follow-up period was 18 months and ranged between 12 months and 20 months.

The inclusion criteria were as follows: (1) one- or double-level lumbar post-discectomy, (2) lumbar spine in- stability grade I or II spondylolisthesis, (3) low back pain (LBP) and/or unilateral sciatica, and (4) failure of conservative treatment for 3 months.

Exclusion criteria were as follows: (1) grade III and IV spondylolisthesis, (2) more than two-level affection, (3) bilateral sciatica, (4) morbid obesity (body mass index $>35 \mathrm{~kg} / \mathrm{m}^{2}$ ), (5) L5-S1 spondylolisthesis with high iliac crest (at the level of L4 pedicles on anteroposterior [AP] lumbosacral X-ray), and (6) major concomitant medical illness.

After ethical committee approval, all patients who met the abovementioned inclusion criteria signed an informed and detailed consent describing the procedure, alternative treatment methods, and possible complications. There were 22 males and eight females with a mean age of 51.8 years (range, 31-70 years). Five eligible patients refused the minimally invasive technique, and they were treated with open PLIF and not included in the study. Complete medical history was obtained, and comprehensive general and neurological examinations were performed.

Preoperatively, plain X-rays of the lumbar spine included AP, lateral, and dynamic flexion and extension views, and a lumbosacral magnetic resonance imaging (MRI). Postoperatively, plain X-rays of the lumbosacral spine were performed at 1, 6, and 12 months. In case of suspected osseous union, computed tomography (CT) of the lumbosacral spine was performed not before 12 months postoperatively or earlier when there was a specific indication, such as suspected loosening of the pedicular screws.

\section{Patient positioning and anesthesia}

All patients were operated under general anesthesia on an appropriately sized spine frame in prone position after cushioning pressure-sensible areas and ensuring a freely hanging abdomen. All patients received $1.5 \mathrm{~g}$ of cefuroxime intravenously 30 minutes before skin incision. Biplanar fluoroscopy was used to locate the affected level. All patients were operated with the same operative technique by the senior author (H.S.) who has 28 years of experience in spine surgery.

\section{Percutaneous pedicular screws insertion}

A $22 \mathrm{G}$ spinal needle was used to verify the appropriate location of the skin incisions. The needle was directly positioned on the skin over the pedicle on an AP image. 
Subsequently, the needle was laterally moved $1.5-3 \mathrm{~cm}$ according to the patients' build and the preoperatively determined pedicular convergence.

A Medtronic PAK (pedicle access kit) Needle (Medtronic, Minneapolis, MN, USA) was used to enter the pedicle. After placing the PAK Needle (Medtronic) at the intersection of the facet and the transverse process, the needle was partially advanced through the pedicle. The inner trocar of the needle was replaced with the guide wire to be inserted into the pedicle. Careful advancement of the guide wire is necessary because this could potentially violate the anterior cortex of the vertebral body.

The fascia and muscle were dilated to allow screw placement by three consecutive dilators. The third dilator served as a tissue protection sleeve during the tapping step, which was performed over the guide wire and fluoroscopic guidance. Screw length was determined using the calibration markings on the shaft of the tap.

Before Spineart Romeo 2 pedicular screws (Spineart SA, Plan-les-Ouates, Switzerland) were inserted into the pedicle, and the screw extenders were assembled to the polyaxial screw and inserted over the guide wire. The process was repeated for the other screws. The screw assemblies should line up and be at approximately the same height outside the patient.

\section{Minimally invasive transforaminal interbody cage application}

A screw-based retractor (Spineart Romeo 2 MIS, Spineart SA) was used, which allowed proper visualization of the disk space and sufficient screw-based distraction. After adjusting the microscope and attaining proper visualization, TLIF and insertion of the appropriate-sized and bone graft-loaded Spineart JULIET Ti transforaminal lumbar cage (Spineart SA) was performed according to the manufacturer's recommendations. By removing the entire ipsilateral facet, the interbody device could be inserted without significant nerve root and thecal sac retraction. Cage filling and disk space filling anterior to the cage was performed by morselized bone obtained from the ipsilateral facet joints (Fig. 1).

After removing the screw-based retractors, appropriately sized rods were inserted and proper compression was applied prior to the final tightening. Wound closure was achieved using 3-0 absorbable interrupted sutures taking the fascia and subcutis as one layer, followed by adhesive

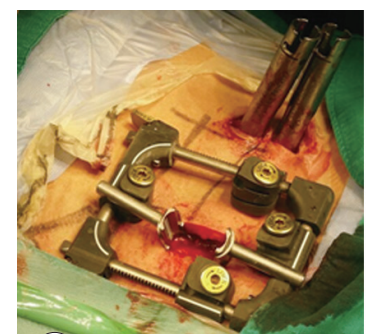

(A)
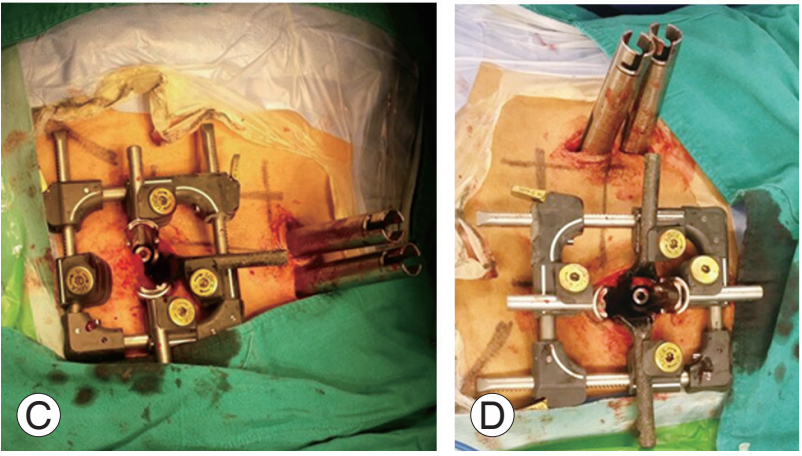

Fig. 1. (A) Application of the screw-based retractor. (B) Distraction. (C) Insertion of the side retractors. (D) Final situation of the situs.

strip application. No surgical drains were used.

Postoperative X-ray was performed, and patients could ambulate on the 1st postoperative day. Separate VAS for limb and back pain were assessed on the day of discharge and after 1, 6, and 12 months.

\section{Outcome measures}

An Arabic version of the ODI questionnaire was applied preoperatively and at each follow-up. Additionally, the VAS was separately used for LBP and radicular leg pain (LP) preoperatively, postoperatively at discharge, and at 1 , 6 , and 12 months postoperatively.

The radiological assessment of fusion was performed according to Ray's criteria [11]: (1) no lucent area around the implant; (2) no fracture of the device, graft, or vertebrae; (3) no sclerotic changes in the graft or adjacent vertebrae; (4) $<3^{\circ}$ of intersegmental position change on dynamic lateral views; and (5) visible bone formation in or around the graft metal.

The minimal clinical important difference (MCID) for ODI and VAS LBP and VAS LP were set in accordance to the study of Parker et al. [12] to ODI 8.2-19.9, VAS LBP 2.2-6.0, and VAS LP 3.9-7.5. Their study specifically determined the MCID after surgical decompression and fusion for the same-level recurrent lumbar stenosis [12]. 


\section{Statistical analysis}

In addition to the standard descriptive statistical analysis, chi-square test for categorical and Student $t$-test for nominal data were used. A repeated measure analysis of variance (ANOVA) for VAS and ODI was conducted, and throughout the study, the significance level and the confidence interval were set to $p=0.05$ and $95 \%$, respectively. Statistical analysis was performed using PASW SPSS Statistics for Windows ver. 18.0 (SPSS Inc., Chicago, IL, USA).

\section{Ethical approval}

All patients were consented in written about the procedure, possible complications, and alternative treatment options prior to participation. Separately, all patients were additionally consented in written to publish their data in a strictly anonymously manner in accordance with international scientific and ethical standards.

\section{Results}

There were 30 patients with 37 lumbar disk levels treated. The most common single levels were L4/L5 and L5/S1 in 20 patients $(66.67 \%)$ and two patients $(6.67 \%)$, respectively. There was one patient (3.33\%) with L3/L4 and seven patients (23.33\%) with a double-level TLIF at L4/L5 and L5/S1. A total of 24 patients ( $80 \%)$ had recurrent disk prolapse; six patients (20\%) had recurrent disk prolapse and spondylolisthesis. Two of the latter (6.67\%) had partial foot drop. Disk re-prolapse occurred on the same side as in the index operation in 20 patients (66.67\%), and all patients received cage implantation from the most symptomatic side. Five patients (16.67\%) had diabetes mellitus; three patients $(10 \%)$ had controlled hypertension; two patients $(6.67 \%)$ had both diabetes and hypertension; and one patient (3.33\%) had diabetes, hypertension, and rheumatoid arthritis (Table 1).

The operative time ranged from 120 to 273 minutes with a mean operative time of 168 minutes. The average blood loss was $386.7 \mathrm{~mL}$ and ranged between $150 \mathrm{~mL}$ and $700 \mathrm{~mL}$. The postoperative hospital stays ranged from 3 to 7 days with an average of 4.3 days (Fig. 2).

The ODI in all patients showed significant improvement from a mean of $73.78 \%$ preoperatively to $16.67 \%$ at 1 month, $15.08 \%$ at 6 months, and $14.13 \%$ at 12 months postoperatively $(p<0.001)$. The preoperative LBP VAS score (mean, 4.37) showed a significant decrease to 1.90 at 1 month, 1.43 at 6 months and 1.10 at 12 months $(p<0.001)$, respectively. Preoperative VAS score of limb pain averaged 7.53 and showed a significant $(p<0.001)$ decrease to a mean of 3.47 at 1 month, 2.43 at 6 months and 1.10 at 12 months. All patients attained radiological fusion at 12 months with 18 (60\%) meeting the Ray's criteria of bony healing at 6-month follow-up (Figs. 3-5).

ANOVA was performed to prove the null hypothesis defined as no change in the ODI, VAS LBP, and VAS

Table 1. Patient's characteristics

\begin{tabular}{lc} 
Characteristic & No. (\%) \\
No. of patients treated & 30 \\
\hline No. of lumbar levels treated & 37 \\
\hline Level L3/4 affection & $1(3.33)$ \\
\hline Level L4/5 affection & 20 (66.67) \\
\hline Level L4/5 and L5/S1 affection & $7(23.33)$ \\
Level L5/S1 affection & 2 (6.67) \\
\hline Same side disc re-herniation & $20(66.67)$ \\
\hline Recurrent disc prolapse & $24(80)$ \\
\hline Recurrent disc prolapse and spondylolisthesis & $6(20)$ with 2 (6.67) having partial foot drop \\
\hline Diabetes mellitus & $5(16.67)$ \\
\hline Controlled hypertension & $3(10)$ \\
\hline Diabetes mellitus and controlled hypertension & 2 (6.67) \\
\hline Diabetes, hypertension, and rheumatoid arthritis & $1(3.33)$ \\
\hline
\end{tabular}



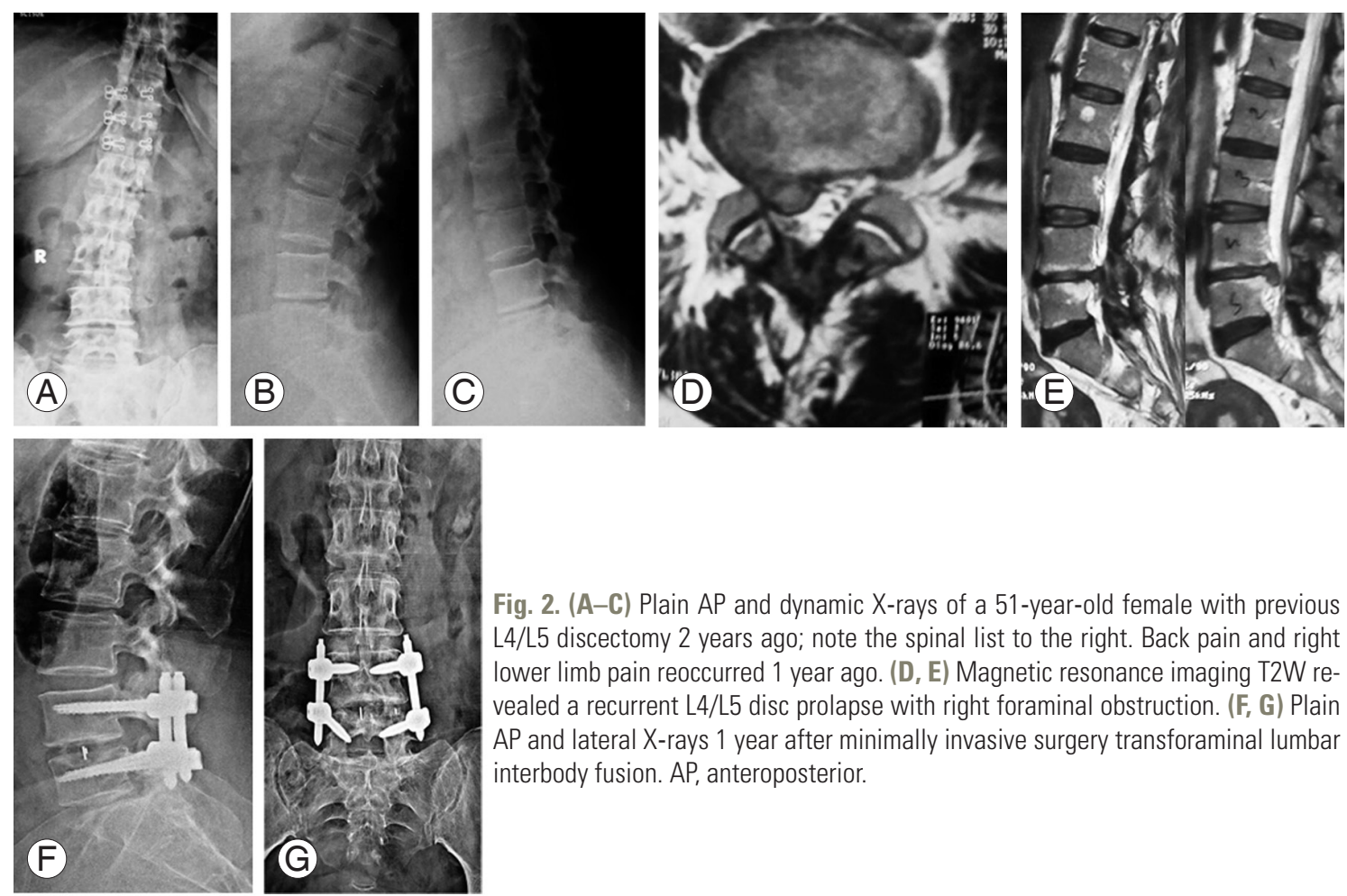

Fig. 2. (A-C) Plain AP and dynamic X-rays of a 51-year-old female with previous L4/L5 discectomy 2 years ago; note the spinal list to the right. Back pain and right lower limb pain reoccurred 1 year ago. (D, E) Magnetic resonance imaging T2W revealed a recurrent $L 4 / L 5$ disc prolapse with right foraminal obstruction. $(\mathbf{F}, \mathbf{G})$ Plain $\mathrm{AP}$ and lateral $\mathrm{X}$-rays 1 year after minimally invasive surgery transforaminal lumbar interbody fusion. AP, anteroposterior.

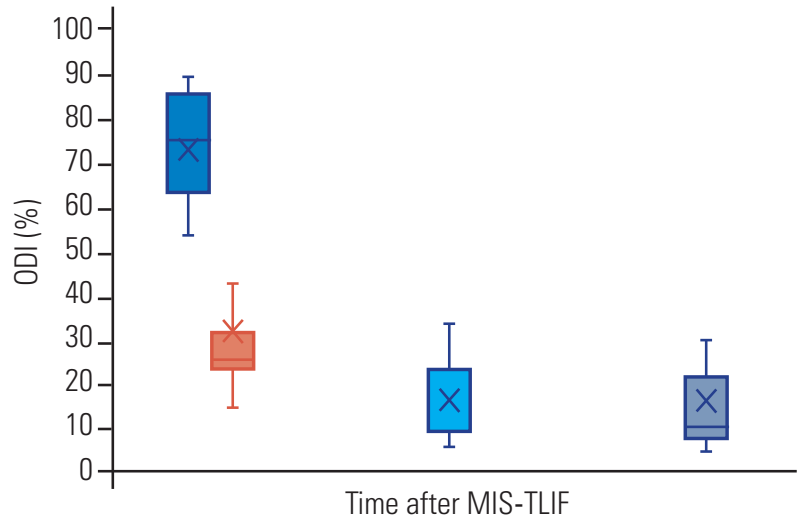

Fig. 3. ODI within the first postoperative year. The middle vertical line equals 6 months postoperatively. Far left depicts the preoperative ODI and far right the ODI at 12 months. ODI, Oswestry Disability Index; MIS-TLIF, minimally invasive surgery transforaminal lumbar interbody fusion.

LP when measured before, 1 month, 6 months, and 12 months after MIS-TLIF, respectively. The results showed a significant time effect for ODI with a Wilks' lambda $=0.5, \mathrm{~F}(3,27), p<0.001, \eta^{2}=0.95$. In addition, VAS LBP (Wilks' lambda=0.381, F[3, 27], $p<0.001, \eta^{2}=0.619$ ) and VAS LP (Wilks' lambda $=0.012, \mathrm{~F}[3,27], p<0.001$, $\eta^{2}=0.988$ ) showed a similar significance. Follow-up comparisons revealed that each pairwise difference was significant in all three measurements $(p<0.01)$. Significant

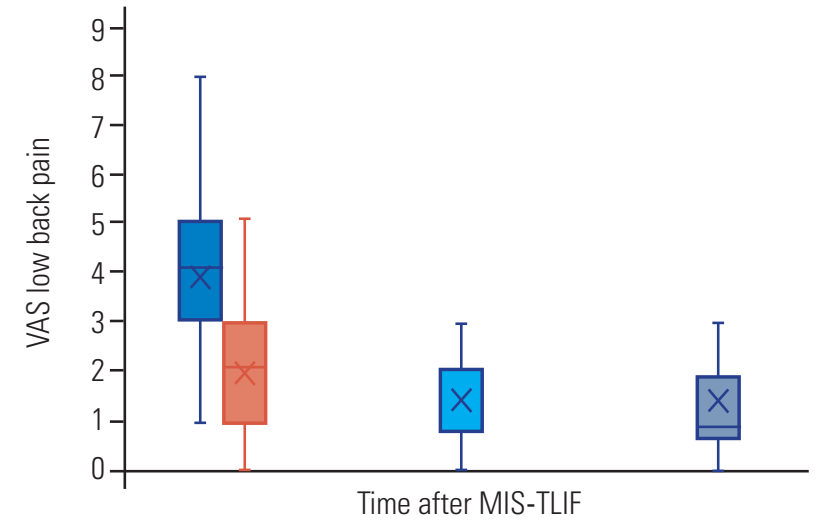

Fig. 4. VAS for lower back pain within the first postoperative year. The middle vertical line equals 6 months postoperatively. Far left depicts the preoperative (VAS low back pain) and far right the (VAS low back pain) at 12 months. VAS, Visual Analog Scale; MIS-TLIF, minimally invasive surgery transforaminal lumbar interbody fusion.

improvement was observed in the respective ODI, VAS LBP, and VAS LP scores over time, suggesting that MISTLIF improved the participants' functional outcome. All patients attained radiological fusion at 12 months with $18(60 \%)$ meeting the Ray's criteria of bony healing at 6-month follow-up (Table 2).

At 12 months, all patients showed improvement considering the set MCID as mentioned above regarding the specific ODI, VAS LBP, and VAS LP. This was further 
verified by the minimal detectable change in MIS-TLIF, considering a standard error of 2.579 for ODI, 0.106 for VAS LBP, and 0.119 for VAS LP, respectively.

No cerebrospinal fluid (CSF) leak, dural tear, screw malpositioning, loosening of screws, or rapidly progressive adjacent segment disease was reported in any case during the follow-up. One patient (3.33\%) with recurrent disk herniation developed contralateral sciatica with unspecific findings in the postoperative MRI that spontaneously improved over the course. Two patients $(6.67 \%$, both double-level TLIFs) received one unit of packed red blood cells postoperatively. Two patients $(6.67 \%)$ developed a superficial infection and were treated uneventfully with antibiotics.

ODI was not statistically related to the preoperative diagnosis of isolated recurrent disk prolapse or recurrent disk prolapse with listhesis $(p>0.05)$. No significant association between preoperative ODI and ODI at 12 months as well as in gender was observed ( $p=0.72$ and $p=0.45$ ), respectively. There was also no significant association

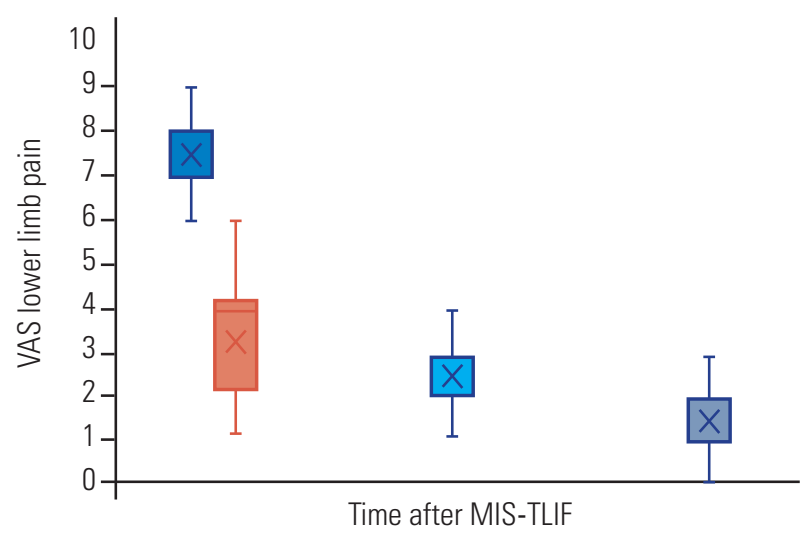

Fig. 5. VAS for limb pain within the first postoperative year. The middle vertical line equals 6 months postoperatively. Far left depicts the preoperative (VAS limb pain) and far right the (VAS limb pain) at 12 months. VAS, Visual Analog Scale; MIS-TLIF, minimally invasive surgery transforaminal lumbar interbody fusion. between preexisting comorbidities and preoperative and postoperative ODI, VAS LBP, and VAS LP with respective $p$-values exceeding 0.05 .

\section{Discussion}

The advent of minimally invasive spine surgery (MISS) marked a new area in contemporary spine surgery because it avoids the approach-related morbidities of traditional open approaches. In revision surgery, conventional surgical spine approaches are associated with prolonged operative time, increased blood loss, paraspinal muscle affection, higher infection rate, and possible neurological deficits $[13,14]$. Revision spine surgery is more challenging than primary surgery because of the unclear anatomical planes and perineural adhesions reflected by a complication rate of $13 \%$ after revision discectomy as reported by Ebeling et al. [1].

Using facetectomy, TLIF allows entrance into previously unharmed virgin tissue avoiding the difficult dissection of fibrotic scar tissues and excessive retraction of scarred nerve roots and dura, minimizing the potential for dural and nerve injuries $[1,15]$. Nevertheless, a higher incidence of dural and nerve injuries was reported with MISS; therefore, this has been challenged by some authors [16]. The restoration of the disc height by the cage indirectly decompresses the neuroforamen thereby improving the leg symptoms [15]. Furthermore, being a $360^{\circ}$ spinal fusion technique, MIS-TLIF is expected to provide higher fusion rates than those using a posterolateral fusion-only technique [17]. All patients in this series showed radiological and clinical fusion at 12 months postoperatively.

The average blood loss of $386.7 \mathrm{~mL}$ was less or comparable to similar studies by El Shazly [18] (653 mL) and Niesche et al. [19]. Blood transfusion was used only in double-level MIS-TLIFs in this series. The duration of MIS-TLIF procedures in our series was comparable to the series by other authors $[8,18,19]$ and evidently prolonged

Table 2. Multivariate tests for ODI, VAS-LBP, and VAS-LP

\begin{tabular}{|c|c|c|c|c|c|c|c|c|}
\hline Variable & $\begin{array}{c}\text { Wilks' lambda } \\
\text { value }\end{array}$ & $F$-value & $\begin{array}{c}\text { Hypothesis } \\
\text { df }\end{array}$ & $\begin{array}{l}\text { Error } \\
\text { df }\end{array}$ & Significance & $\begin{array}{c}\text { Partial eta } \\
\text { squared }\end{array}$ & $\begin{array}{c}\text { Noncentrality } \\
\text { parameter }\end{array}$ & $\begin{array}{c}\text { Observed } \\
\text { power }^{\text {a) }}\end{array}$ \\
\hline Multivariate tests ODI & 0.050 & $169.488^{b)}$ & 3.000 & 27.000 & 0.000 & 0.950 & 508.464 & 1.000 \\
\hline Multivariate tests VAS-LBP & 0.381 & $14.594^{\mathrm{bl}}$ & 3.000 & 27.000 & 0.000 & 0.619 & 43.783 & 1.000 \\
\hline Multivariate tests VAS-LP & 0.381 & $14.594^{\mathrm{bl}}$ & 3.000 & 27.000 & 0.000 & 0.619 & 43.783 & 1.000 \\
\hline
\end{tabular}

ODI, Oswestry Disability Index; VAS, Visual Analog Scale; LBP, low back pain; LP, leg pain; df, degrees of freedom.

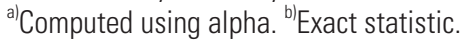


in double-level MIS-TLIFs. Hospital stay averaged 4.3 days in this series and was comparable to a study of $\mathrm{Ni}$ esche et al. [19].

The present study verifies the observation of other authors that ODI, VAS for LBP, and VAS for lower limb pain significantly decreased during the early postoperative phase and continued to improve within the first year [18-21]. Two patients (6.67\%) had superficial wound infections conservatively treated by sensitive antibiotics, and one patient (3.33\%) had a contralateral radiculopathy without proper radiological signs on postoperative MRI and CT, although the patient showed spontaneous improvement within the first year. In 2014, Niesche et al. [19] reported that a total of four of 43 patients $(9.30 \%)$ required surgical wound debridement; of these four, two patients (4.65\%) showed postoperative neurological deterioration.

In their series comprising 54 patients with post-discectomy pain syndrome, Erdem et al. [22] found MIS-TLIF to be an efficient technique for post-discectomy pain that is resistant to conservative treatment options as well as for patients who had a second re-herniation. A total of 27 patients underwent MIS-TLIF and when compared to the 15 patients who underwent re-discectomy and seven who were treated by nonsurgical means had a better VAS and ODI at final follow-up [22].

Ganesan et al. [23] compared a modified mini-open TLIF technique with a conventional open TLIF technique in their series of 51 patients and concluded that there was a significant reduction in blood loss, hospital stay, and postoperative back pain. However, at 2-year follow-up, there was no discrepancy regarding clinical and radiological outcome parameters. Wu et al. [24] compared minimally invasive TLIF to open TLIF in 167 patients with grade II or less spondylolisthesis and verified a similar superiority of minimally invasive TLIF regarding blood loss and postoperative hospital stay, but no difference in the functional and radiological outcomes.

Elmekaty et al. [25] compared in a series of 59 patients with single-level lumbar spondylolisthesis MIS-posterolateral fusion, MIS-transforaminal lumbar interbody fusion (MIS-TLIF), and midline lumbar fusion (MIDLF) and concluded that MIDLF to be the least invasive. Furthermore, there was no difference regarding fusion and functional outcomes.

A total of 43 patients were treated with open TLIF in a series by Chen et al. [26]. Two patients (4.65\%) had a dural tear, and one patient (2.33\%) had a superficial infection. In 2013, El Shazly et al. [18] reported on 15 patients treated only with re-discectomy without fusion and another group of 15 patients treated with discectomy and TLIF. In the first group, there was one disc re-prolapse (6.67\%), one postoperative instability (6.67\%), four dural tears $(26.67 \%)$, and two neurological deteriorations (13.33\%). In the TLIF group, two patients experienced dural tears (13.33\%), one patient (6.67\%) developed deep venous thrombosis, and one experienced neurological worsening $(6.67 \%)$ [18].

In 2013, Sonmez et al. [27] reported on 20 patients; 10 underwent MIS-TLIF with percutaneous unilateral pedicular instrumentation and the other 10 underwent MISTLIF with bilateral percutaneous pedicular instrumentation. The fusion rates reached $80 \%$ and $90 \%$ in the first and second group, respectively.

In 2014, Lequin et al. [28] treated 26 patients with recurrent disc herniations with stand-alone trabecular metal cages in the PLIF technique. They reported that four patients (15\%) underwent reoperation, two due to a postoperative hematoma, one for persistent CSF leakage, and one for recurrent severe back pain associated with instability and lucency around the cage. Based on a series of 73 patients, Li et al. [29] concluded that open TLIF is an effective, reliable, and safe alternative procedure for the treatment of recurrent lumbar disc herniation.

In 2015, Jin-Tao et al. [30] found a similar fusion and complication rate for both open and MIS-TLIF. Moreover, the MIS-TLIF group tended to have a higher revision/ readmission rate that might be associated with the deep learning curve.

Lee et al. [21] compared open versus MIS-TLIF in 144 patients and had similar clinical and radiological outcomes when compared to this study and emphasized the advantages of MIS-TLIF, particularly, less perioperative blood loss and pain, earlier rehabilitation, and a shorter hospital stay.

The versatility of MIS-TLIF was shown by HansenAlgenstaedt et al. [31] when they described a minimally invasive revision of adjacent-level disease after MIS deformity surgery.

Some of the limitations of the study are the small patient cohort, single-center, and single-surgeon nature in addition to single-implant and application system of this study. Thus, larger level I or II multicentric studies are required in future. 


\section{Conclusions}

MIS-TLIF offers a valid, safe, effective, and reproducible treatment option for patients with lumbar post-discectomy syndromes.

\section{Conflict of Interest}

No potential conflict of interest relevant to this article was reported.

\section{Author Contributions}

ABA: data collection, surgical assistance, manuscript writing, statistics; AYA: data collection, surgical assistance, literature research; AMS: data collection, literature research, manuscript writing, statistics; ASB: literature research, manuscript writing, editing; WK: idea, supervision, proof reading; YE: idea, supervision, proof reading; and HS: idea, surgeon, proof reading.

\section{References}

1. Ebeling U, Kalbarcyk H, Reulen HJ. Microsurgical reoperation following lumbar disc surgery: timing, surgical findings, and outcome in 92 patients. J Neurosurg 1989;70:397-404.

2. North RB, Campbell JN, James CS, et al. Failed back surgery syndrome: 5-year follow-up in 102 patients undergoing repeated operation. Neurosurgery 1991;28:685-91.

3. Inoue S, Kamiya M, Nishihara M, Arai YP, Ikemoto T, Ushida T. Prevalence, characteristics, and burden of failed back surgery syndrome: the influence of various residual symptoms on patient satisfaction and quality of life as assessed by a nationwide Internet survey in Japan. J Pain Res 2017;10:811-23.

4. Resnick DK, Choudhri TF, Dailey AT, et al. Guidelines for the performance of fusion procedures for degenerative disease of the lumbar spine: part 8: lumbar fusion for disc herniation and radiculopathy. J Neurosurg Spine 2005;2:673-8.

5. Bettini N, Girardo M, Dema E, Cervellati S. Evaluation of conservative treatment of non specific spondylodiscitis. Eur Spine J 2009;18 Suppl 1:143-50.

6. Ganty P, Sharma M. Failed back surgery syndrome: a suggested algorithm of care. Br J Pain 2012;6:153-61.
7. Berjano P, Pejrona M, Damilano M. Microdiscectomy for recurrent L5-S1 disc herniation. Eur Spine J 2013;22:2915-7.

8. Niemeyer T, Halm H, Hackenberg L, Liljenqvist U, Bovingloh AS. Post-discectomy syndrome treated with lumbar interbody fusion. Int Orthop 2006;30:163-6.

9. Archavlis E, Carvi y Nievas M. Comparison of minimally invasive fusion and instrumentation versus open surgery for severe stenotic spondylolisthesis with high-grade facet joint osteoarthritis. Eur Spine J 2013;22:1731-40.

10. McAfee PC, Phillips FM, Andersson G, et al. Minimally invasive spine surgery. Spine (Phila Pa 1976) 2010;35(26 Suppl):S271-3.

11. Ray CD. Threaded fusion cages for lumbar interbody fusions: an economic comparison with 360 degrees fusions. Spine (Phila Pa 1976) 1997;22:681-5.

12. Parker SL, Mendenhall SK, Shau DN, et al. Minimum clinically important difference in pain, disability, and quality of life after neural decompression and fusion for same-level recurrent lumbar stenosis: understanding clinical versus statistical significance. J Neurosurg Spine 2012;16:471-8.

13. O’Toole JE, Eichholz KM, Fessler RG. Surgical site infection rates after minimally invasive spinal surgery. J Neurosurg Spine 2009;11:471-6.

14. Gadjradj PS, van Tulder MW, Dirven CM, Peul WC, Harhangi BS. Clinical outcomes after percutaneous transforaminal endoscopic discectomy for lumbar disc herniation: a prospective case series. Neurosurg Focus 2016;40:E3.

15. Abd El-Kader Hel-B. Transforaminal lumbar interbody fusion for management of recurrent lumbar disc herniation. Asian Spine J 2016;10:52-8.

16. Li X, Han Y, Di Z, et al. Percutaneous endoscopic lumbar discectomy for lumbar disc herniation. J Clin Neurosci 2016;33:19-27.

17. Mobbs RJ, Phan K, Malham G, Seex K, Rao PJ. Lumbar interbody fusion: techniques, indications and comparison of interbody fusion options including PLIF, TLIF, MI-TLIF, OLIF/ATP, LLIF and ALIF. J Spine Surg 2015;1:2-18.

18. El Shazly AA, El Wardany MA, Morsi AM. Recurrent lumbar disc herniation: a prospective comparative study of three surgical management procedures. Asian J Neurosurg 2013;8:139-46. 
19. Niesche M, Juratli TA, Sitoci KH, et al. Percutaneous pedicle screw and rod fixation with TLIF in a series of 14 patients with recurrent lumbar disc herniation. Clin Neurol Neurosurg 2014;124:25-31.

20. Hlubek RJ, Mundis GM Jr. Treatment for recurrent lumbar disc herniation. Curr Rev Musculoskelet Med 2017;10:517-20.

21. Lee KH, Yue WM, Yeo W, Soeharno H, Tan SB. Clinical and radiological outcomes of open versus minimally invasive transforaminal lumbar interbody fusion. Eur Spine J 2012;21:2265-70.

22. Erdem MN, Erken HY, Aydogan M. The effectiveness of non-surgical treatments, re-discectomy and minimally invasive transforaminal lumbar interbody fusion in post-discectomy pain syndrome. J Spine Surg 2018;4:414-22.

23. Ganesan S, Jayabalan V, Kumar V, Kailash K. Clinical and radiological outcomes of modified mini-open and open transforaminal lumbar interbody fusion: a comparative study. Asian Spine J 2018;12:544-50.

24. Wu AM, Hu ZC, Li XB, et al. Comparison of minimally invasive and open transforaminal lumbar interbody fusion in the treatment of single segmental lumbar spondylolisthesis: minimum two-year follow up. Ann Transl Med 2018;6:105.

25. Elmekaty M, Kotani Y, Mehy EE, et al. Clinical and radiological comparison between three different minimally invasive surgical fusion techniques for single-level lumbar isthmic and degenerative spondylolisthesis: minimally invasive surgical posterolat- eral fusion versus minimally invasive surgical transforaminal lumbar interbody fusion versus midline lumbar fusion. Asian Spine J 2018;12:870-9.

26. Chen Z, Zhao J, Liu A, Yuan J, Li Z. Surgical treatment of recurrent lumbar disc herniation by transforaminal lumbar interbody fusion. Int Orthop 2009;33:197-201.

27. Sonmez E, Coven I, Sahinturk F, Yilmaz C, Caner H. Unilateral percutaneous pedicle screw instrumentation with minimally invasive TLIF for the treatment of recurrent lumbar disk disease: 2 years follow-up. Turk Neurosurg 2013;23:372-8.

28. Lequin MB, Verbaan D, Bouma GJ. Posterior lumbar interbody fusion with stand-alone trabecular metal cages for repeatedly recurrent lumbar disc herniation and back pain. J Neurosurg Spine 2014;20:617-22.

29. Li Z, Tang J, Hou S, et al. Four-year follow-up results of transforaminal lumbar interbody fusion as revision surgery for recurrent lumbar disc herniation after conventional discectomy. J Clin Neurosci 2015;22:331-7.

30. Jin-Tao Q, Yu T, Mei W, et al. Comparison of MIS vs. open PLIF/TLIF with regard to clinical improvement, fusion rate, and incidence of major complication: a meta-analysis. Eur Spine J 2015;24:1058-65.

31. Hansen-Algenstaedt N, Liem M, Khalifah SO, Holz J, Giese A. Minimal-invasive revision of adjacent level disease after MIS deformity surgery. Eur Spine J 2017;26(Suppl 5):562-4. 